\title{
Adapting a component-based model approach to SOA: A robotic experience
}

\author{
Francisca Rosique ${ }^{1}$, Nour Ali ${ }^{2}$ and Fernando Losilla ${ }^{1}$ \\ ${ }^{1}$ Universidad Politécnica de Cartagena, Spain \\ ${ }^{2}$ Brunel University London, United Kingdom \\ \{paqui.rosique,fernando.losilla\}@upct.es,nour.ali@brunel.ac.uk
}

\begin{abstract}
Keywords: $\quad$ Real Time (RT), Service Component Architecture (SCA), Service Oriented Architecture (SOA), Component Based Software Engineering (CBSE), Model Driven Software Development (MDSD), Robotic Systems.

Abstract: $\quad$ C-Forge is an approach that combines Component Based Software Engineering (CBSE) and Model Driven Software Development (MDSD), and has been previously used to define the software architecture of robotic systems. However, as robotic systems become part of a dynamic and heterogeneous environment, CBSE becomes limited. A paradigm that promises to easily adapt and integrate collaborative, heterogeneous and distributed systems is Service Oriented Architecture (SOA). In this paper, we enrich C-Forge with service oriented architectural primitives by extending its CBSE metamodel and Model Driven Methodology.
\end{abstract}

\section{INTRODUCTION}

Technological advances in multiple disciplines such as telecommunications and informatics have contributed to the emergence of reactive systems, especially in the robotic domain. Besides, improvements in network technologies, particularly wireless networking, have revolutionized how robots perceive the environment and interact with it. Robots can now be connected to different devices such as mobile phones or sensors, which allows them to react in real time to events of the rest of the system. For example, a robot can be controlled remotely by a mobile user or it can detect an obstacle and recalculate its path. Robots can also collaborate in real time, responding to other robots' tasks such as movements, trajectories, etc.

These systems have to dynamically integrate and perform collaborative tasks with diverse devices and software systems. This change in the nature of robotic systems enforces the usage of new software engineering paradigms for their development and evolution. New developments should provide for (1) integration between heterogeneous systems, (2) independence of location, implementation and usage, (3) reusability, (4) integration with reactive environments, (5) real time.
The Division of Electronics Engineering and Systems (División de Sistemas e Ingeniería Electrónica, DSIE) Research Group has a long experience in developing software for services robots, applying different approaches (reference architectures, Component-based software engineering (CBSE), and Model Driven Software Development (MDSD)) (Diego et al., 2010) to cope with the increasing complexity of new projects since 1999. The DSIE has recently developed C-Forge (CForge, 2015), an Eclipse based Model Driven toolchain for supporting a component based development process. C-Forge has been used in the last years to implement hybrid robotic architectures by defining a set of static components that interact with each other though their ports. It is remarkable the flexibility and ease to change, at design time, the kind of components, its ports or interaction interfaces, as well as the connections between them and their distribution in different processes, nodes or concurrent regions. An example of the application of C-Forge to an Autonomous Underwater Vehicle is described in (Ortiz et al., 2015).

However, this flexibility in design time is not the same in run-time. In our experience, there are certain components that require a static and stable connection. But as we reach the higher layers, where components that require some intelligence are, for 
example a mission planner, it is highly recommended to have a reconfiguration capability comparable to the variability that may occur in the mission. To adapt to this situations, two possible ways were detected:

1. Dynamic reconfiguration, where new links between components are created when necessary, for instance when performing system reconfiguration, which involves the replacement of components or links. However, this may not be the optimal solution if we just want to use temporarily the services offered by other component.

2. Use Service Oriented Architecture (SOA) to allow components to look for the run-time available services that they can use, as well as to publish their services so that other components can use them.

In this context, SOA is a paradigm that, even though is not new, it is becoming increasingly important as a solution to some of the new requirements that society imposes on robotic systems. SOA (Erl, 2008) is especially useful for providing a solution to the integration, implementation and location independence of systems. Therefore, we decided to experiment with a mixed structure where the components themselves offer services as SOA architectures. For example, following the case study presented in (Ortiz et al., 2015), a mission planner component would create new missions depending on the available services at the time. In this way, the aim is to combine the power of a CBSE-MDSD process with the flexibility of SOA. For this purpose, it is necessary to extend C-Forge (that initially only considers a pure CBSE process) so that components can publish their services and use the services published by others. In this way, when a component needs to discover the available services, assuming that they may change, it could use the services that have been published by the other components that are included in the architecture.

The rest of this paper is organized as follows: Section 2 introduces C-Forge, our previous experience which has motivated this work. Section 3 describes the SOA adaptation process into C-Forge and finally Section 4 presents the conclusions and further work.

\section{C-FORGE}

C-Forge is a tool-chain developed with the Eclipse environment that uses its Model Driven Software Development (MDSD) plugins to provide support for developing component based applications (Rosique et al., 2016). C-Forge consists of the following tools: (1) a language to model component based applications, called WCOMM, (2) a framework called FraCC, which provides run-time support for the applications modeled using WCOMM.

\subsection{WCOMM component language}

A WCOMM component is an entity that encapsulates its internal state and comprises both structural and behavioral parts. The structural part is defined by its ports and the messages that flow through them, grouped in interfaces. These messages are sent following the asynchronous no-reply communication scheme. Behaviour is defined by means of a finite state machine, similar to the defined in UML, extended with temporal properties. That is, the user models the behaviour of the component by means of states, transitions, events, guards and orthogonal and hierarchical regions. Each state can have additionaly an internal activity, which will be later associated with code in FraCC. WCOMM also models what we called the the "shell" of the activity, formed by the messages that are exchanged and the events that are created. These events, along with the reception of messages through ports, are responsible for the change of the component state. Therefore, they establish the connection between structure and behavior. Finally, an application is modeled as a set of components interconnected among them.

\subsection{Framework FraCC}

FraCC is a component based framework implemented in $\mathrm{C}++$ that was developed with the purpose of providing (1) full support to the characteristics of the WCOMM component model, (2) full control over the concurrency characteristics of the application, letting the user decide how many processes and threads will be created and in which threads the components will run and (3) explicit control of the assignment of components to computational nodes. These features allow the use of FraCC in applications with real-time constraints.

\section{ADAPTING C-FORGE TO SOA}

This section briefly describes the main characteristics of the adaptation process. The first step is adapting our metamodel, which extends the C-Forge metamodel (see Figure 1) in order to support SOA constructs. The second step is to establish the work methodology. 


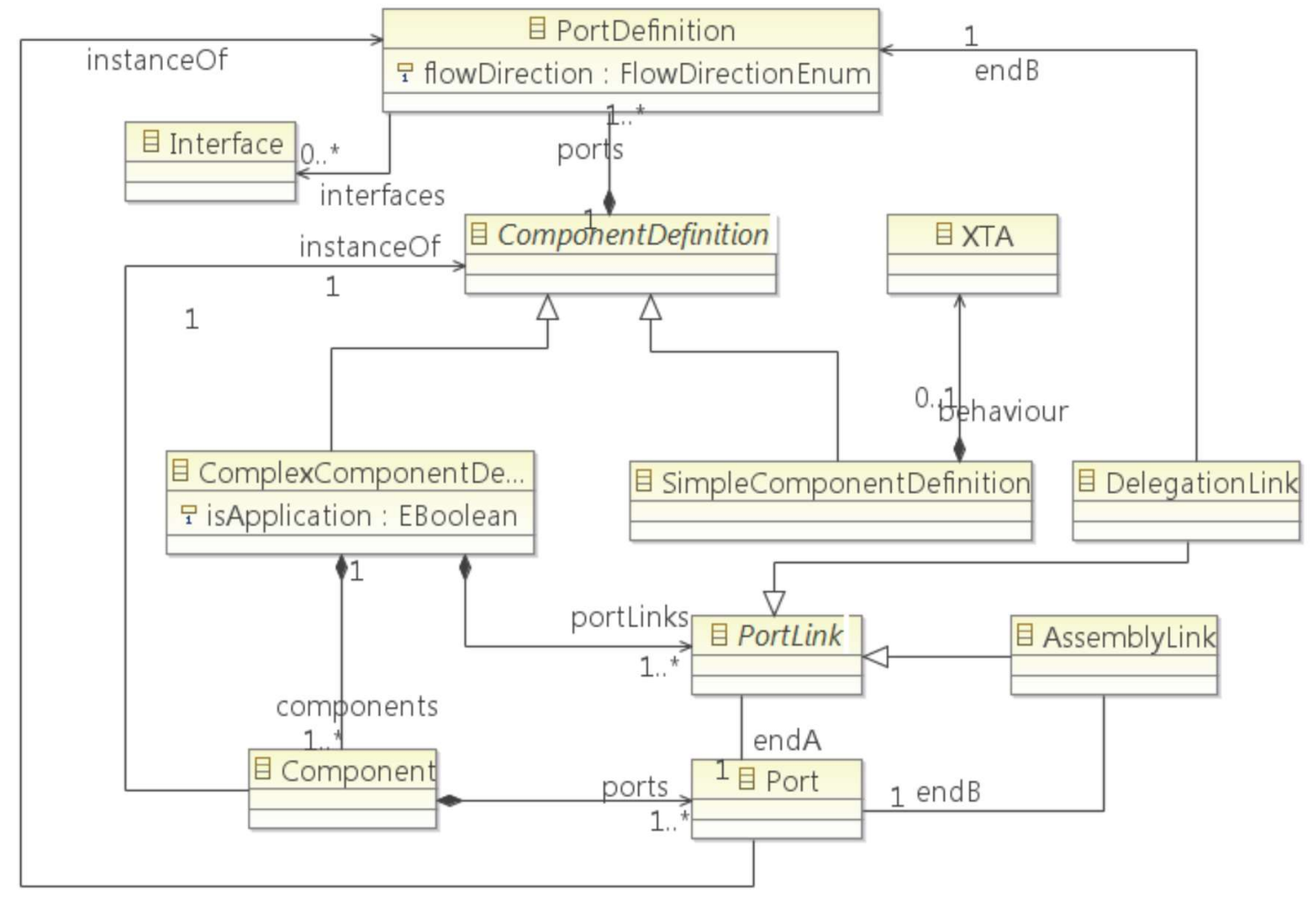

Figure 1: Excerpt of WCOMM metamodel

\subsection{SOA Extended Metamodel}

The main idea of the proposal is to integrate SOA (Ali and Babar, 2009) characteristic concepts by making use of the previously defined C-Forge artifacts. Among the most important concepts of the proposal are Contract and Choreography. In SOA, services adhere to a communication agreement, which is defined along with one or more service description documents. This contract is usually represented by a sequence diagram. Would it be possible to represent this contract with other models or diagrams? Our proposal relies on being able to take advantage of the elements of the structural notation of the component model to define this contract, more specifically, making use of state machines.

In this regard, the SOA concepts as well as the concepts of the metamodel of the WCOMM component language (see Figure 1) proposed previously have been thoroughly studied. Similar concepts have been found in both approaches (services, interfaces, ports, etc.). Consequently, the possibility of defining a SOA architecture from a WCCOM component model that interrelates services by means of interfaces and well-defined contracts between these services has been devised. Interfaces are defined in a neutral way that must be independent from the hardware platform, the operating system and the programming language in which the service is implemented. This fact allows services, built on different systems, to interact among them in a uniform and universal way.

Figure 2 shows, in red color, the SOA concepts that have been added to C-Forge: Contract, Choreography, EndPoint, Interface, and ServiceChannel.

The elements involved in this proposal are:

- Component. It retains the elements of the structural notation defined in our previous work, differentiating definition from instances for reutilization purposes. This fact can be appreciated in the ComponentDefinition and Component concepts. The Component concept is directly related with the SOA concept of 
Participants and allows to define the service providers and consumers.

- Interface. This concept is common to both approaches. It describes the operations used between a service provider and a service consumer from the perspective of the provider.

- EndPoint. Components (Participants) provide or consume services via the EndPoints. These EndPoints have a direct correspondence to what in the original WCOMM metamodel was called Port. An EndPoint is the part or feature of a component which acts as the interaction point for a service - where it is provided or consumed. When an EndPoint is a provider it contains at least a ServicePoint. When an EndPoint is a consumer it contains at least a RequestPoint.

- ServicePoint. A ServicePoint defines a capability offered by one entity to others.

- RequestPoint. A RequestPoint defines the connection point through which a Participant makes requests or consumes services. A participant can be a consumer, a provider or both.

- ServiceChannel. ServiceChannel provide a communication path between consumer (RequestsPoint) and provider services (ServicePoint).

- Contract define the terms, conditions, interfaces and choreography that participants must agree to. They specify how services are provided and consumed based on interactions and behaviors involving the participants
(Components). Each role or party involved in a Contract is defined by an Interface or Interfaces, which denotes the type of the role. A Contract is a binding contract - binding on any participant that has a service port typed by a role (EndPoint) in a service contract. It defines the relationships between a set of roles defined by Interfaces.

- Choreography. An important part of the Contract is the choreography. The choreography is a specification of what is transmitted and when it is transmitted between parties to enact a service exchange. The choreography specifies exchanges between the parties - the data, assets and obligations between the parties. The choreography defines what happens between the provider and consumer participants without defining their internal processes - their internal processes do have to be compatible with their Contracts. A Contract Choreography is a diagram behavior usually defined by an interaction diagram or activity diagram. In this approach the choreography is specified by an Extended Timed Automata (extended state machine) represented as XTA element in the metamodel.

- ServiceArchitecture. It consists in the interaction of different service provider and consumer roles (contracts and participants) to achieve a goal. Because of this, a collaboration model is used to represent it.

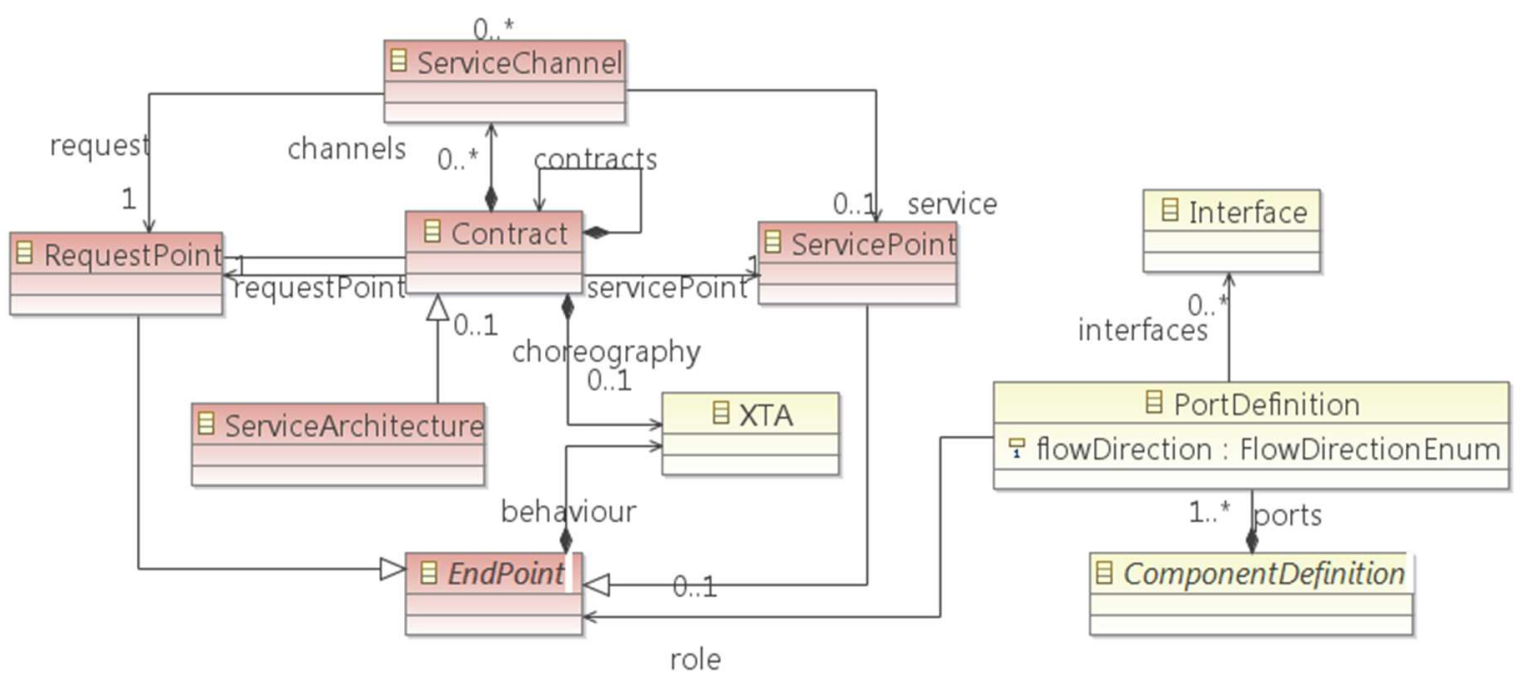

Figure 2: Excerpt of metamodel, focused on its SOA concepts (elements highlighted in red). 


\subsection{Work Methodology: case study}

In order to demonstrate the new proposal, a simple case study based on an oceanographic system has been carried out. The system is composed of a series of smart buoys deployed in a marine environment, a small AUV (Autonomous Underwater Vehicle) and a landside central control office in charge of managing and planning the missions of the AUV. The main mission of the AUV consists in traversing the seafloor gathering data and sending them to the central office. Buoys also collect and send data. In addition, they also send emergency reports when necessary (atmospheric and maritime phenomena, etc.). Finally, the central office receives all the data in real time and, whenever it is necessary, it sends a mission update command to the UAV (go back to the meeting point, change a route in search of new data, etc.).

Considering this study case, we will perform some steps that will allow us to devise the architecture of the system. When creating a ServicesArchitecture by using the top-down design approach, it is important to think about the problem that is being solved or what you are attempting to accomplish. The steps are the following:

1. Identify the mission/goal of the robotic system. In this first step, the main mission of the system must be identified and assigned as "ServiceArchitecture". In our study case, the main goal of the system is underwater marine inspection and this goal is used as the name of the "ServiceArchitecture" (Underwater Marine Inspection), as can be seen in the upper part of Figure 3. In the following steps, the next roles involved in the architecture (ServiceContract and Participants) will be identified, as well as the composite services in which these roles participate.

2. Identify Participants of the Architecture. In this step the participants (components) are added to the architecture. These are represented as parts or roles in the service architecture. We choose a set of components from our initial component based architecture as participants. Participants are components that participate in the service architecture providing/requiring services. In our case, they are: "UAV", "Buoyl/ Buoy $2 / .$. BuoyN/" and "MissionInterface". These components correspond to complex highlevel components but, in order not to increase the complexity of the case study, we will not go into more detail with them (the component diagram corresponding to the UAV component in this example can be seen with greater detail in (Ortiz et al., 2015)).

3. Define RequestPoint and ServicePoint. Once the participants are identified, it is necessary to define which EndPoints (ports) are involved in the architecture. This is required in order to be able to define a Contract in the following steps. It is also necessary to indicate the type of the EndPoints (RequestPoint or ServicePoint). The more precise this step is, the easier it will be to define the contract. To this effect, step 3 must be complemented by step 4. The EndPoints used in the case study are "PlanCmd", "ReportSts", "EmergencyCall" y "DataSts". In Figure 3 it can be observed how they are distributed among the different participants.

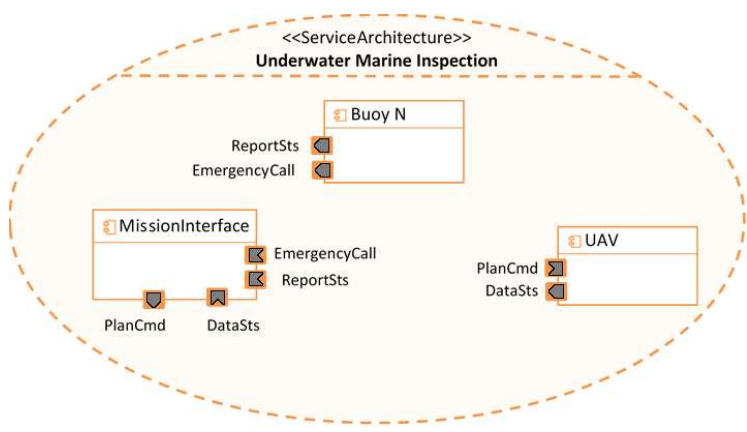

Figure 3: Architecture, participants and endpoints definition.

4. Define Service Interfaces of the EndPoint. In this step the ServiceInterfaces of each component are defined.

5. Specify Service Contract. We define the service contract from the point of view of the service providers. The service contracts define the roles of the service provider and service consumer. The service provider indicates the choreography of the messages and the rules for the provision of a service that the consumer has to fulfil.

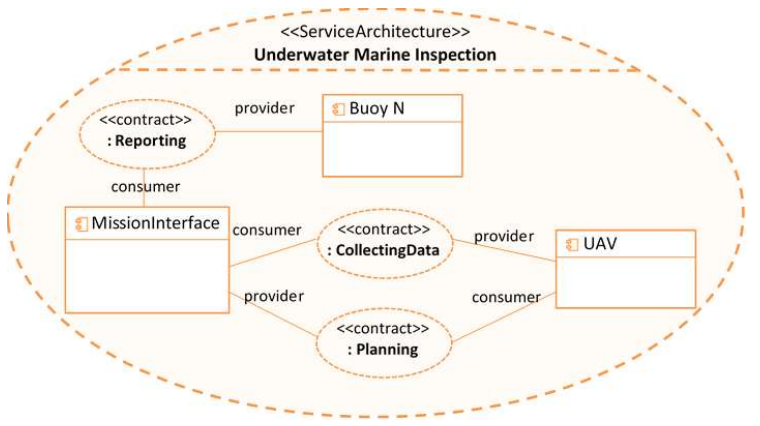

Figure 4: Architecture view including contracts and participants. 


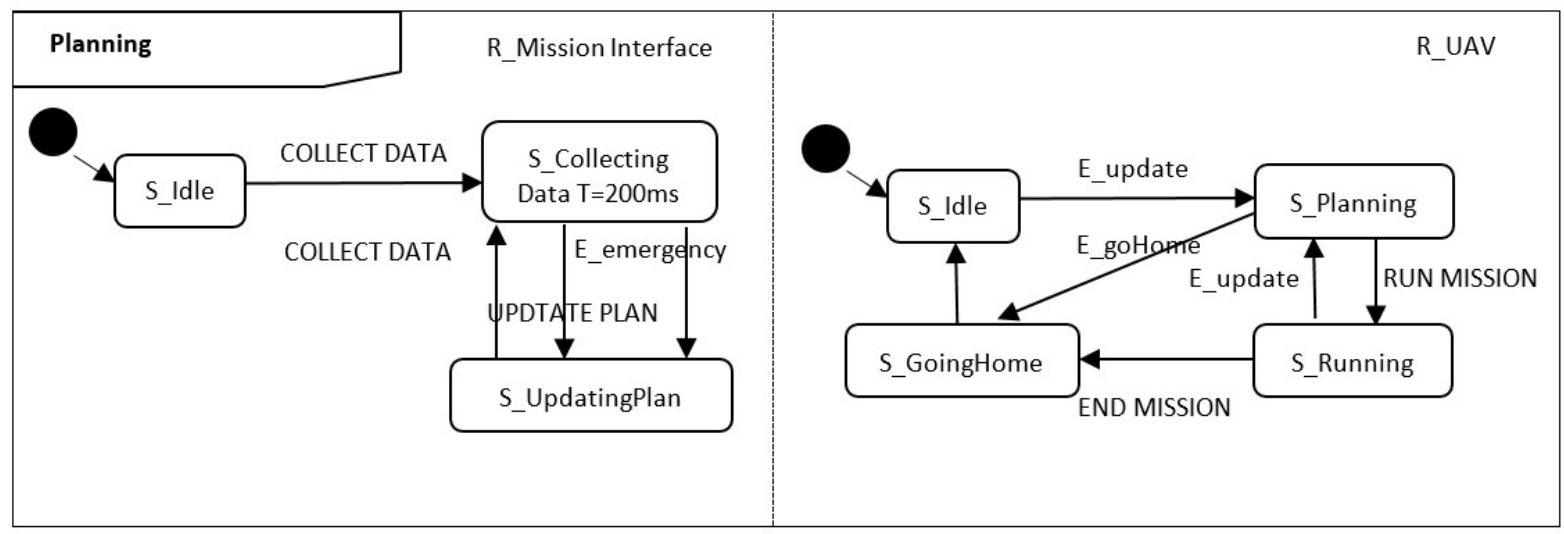

Figure 5: Choreography of the Planning contract.

6. Specify Choreography. This is one of the most important steps in this new approach, since it includes its main novelty, the use of state machines instead of sequence diagrams. To accomplish this step several sub-steps must be taken:
a. A state machine will be created for each defined contract.
b. A concurrent region of the state machine will be implemented for each participant involved in the contract. They will run in parallel and in real time.
c. A state machine will be added to each region, where interfaces involved in the contract will correspond to transition events that trigger state changes. Inside each state, activities generating events can be run. In Figure 5, the state machine corresponding to the Planning Service Contract can be seen.

\section{CONCLUSIONS}

In this paper, we have proposed the combined use of SOA, MDSD and CBSE and we have presented a methodological guide that allows us to integrate the SOA process in the C-Forge development process. In this regard, the proposal of using state machines to define the choreography is a feasible option to adapt our component system to SOA in a simple way. Future work includes developing a framework that includes the research challenges discussed in this paper (architectural modelling, mappings, monitoring).

\section{ACKNOWLEDGEMENTS}

This research has been supported by the MINECO/FEDER project grant TEC2016-76465C2-1-R (AIM), DGT (ref. SPIP2017-02286) and the "Research Program for Groups of Scientific Excellence in the Region of Murcia" of the Seneca Foundation (Agency for Science and Technology in the Region of Murcia - 19895/GERM/15), Spain.

\section{REFERENCES}

Ali, N., Babar, M.A., 2009. Modeling Service Oriented Architectures of Mobile Applications by Extending SoaML with Ambients, in: 2009 35th Euromicro Conference on Software Engineering and Advanced Applications.,

C-Forge | Eclipse-based model-driven tool-chain for supporting a component-based development [WWW Document], 2015. C-Forge. URL http://www.dsie.upct.es/cforge/ (accessed 3.12.18).

Diego, A., Cristina, V.-C., Francisco, O., Juan, P., Bárbara, Á., 2010. V3cmm: A 3-view component meta-model for model-driven robotic software development. J. Softw. Eng. Robot. 1, 3-17.

Erl, T., 2008. Soa: principles of service design. Prentice Hall Upper Saddle River.

Ortiz, F.J., Insaurralde, C.C., Alonso, D., Sánchez, F., Petillot, Y.R., 2015. Model-driven analysis and design for software development of autonomous underwater vehicles. Robotica 33, 1731-1750. https://doi.org/10.1017/S0263574714001027

Rosique, F., Alonso, D., Ortiz, F., 2016. Modeling and Executing Component-based Applications in C-Forge, in: 2016 11th International Conference on Software Technologies (ICSOFT). 